\section{The role of thromboelastography (TEG) in postoperative bleeding in patients undergoing Coronary Artery Bypass Grafting (CABG) with Cardiopulmonary Bypass (CPB).}

Spyridakis Emmanouil, , Chatzieleftheriou Nikolaos, Pendilas Nikolaos,

Eleni Pappa, Kalakonas Spyros

"Hygeia" General Hospital

Athens, Greece

\section{(7) hygeia}

\section{Background}

Bleeding in cardiothoracic surgery is often multifactorial. Postoperative hemorrhage results in longer length of hospital stay, higher mortality rates and higher healthcare costs. Thromboelastography (TEG) measures viscoelastic properties of clot formation and is currently best known for perioperative management in order to decrease blood transfusion and optimize the administration of blood products in cardiac surgery.

The purpose of this study is to examine the immediate postoperative thromboelastography (TEG) results of patients who had bled after CABG with $C P B$ and demonstrate its predictive value on postoperative hemorrhage.

\section{Material and Methods}

We retrospectively analyzed 20 patients with excessive postoperative bleeding with or without chest re-exploration. Blood loss was measured by calculating the volume of mediastinal and pleural drainage for the first 18 hours. Excessive bleeding was defined as $>1000 \mathrm{ml}$ over 18 hours. Baseline kaolin-activated TEG was performed before the induction of anaesthesia and one just before the transportation to the ICU, using the same TEG coagulation analyser, the same personnel, and according to manufacturers guidelines. The TEG parameters analyzed_were: $R$ value (reaction time), $\mathrm{K}$ value, $\mathrm{A}$ angle, MA (Maximum Amplitude) , $G$ value, $\mathrm{Cl}$ (coagulation index) and $L Y 30$ index.

\section{$\underline{\text { Results }}$}

The preanesthesia TEG was normal at $60 \%$ of patients and hypercoagulable at $\mathbf{4 0} \%$ of the patients included.

Prior to ICU departure, $\mathbf{2 5 \%}$ of bleeding patients had normal TEG , $65 \%$ had shown hypercoagulability and $10 \%$ had shown bleeding tendency based on TEG measurements. All investigated patients experienced severe bleeding and $10 \%$ needed surgical reopening. (Table 1)
In our study, neither the normal nor the hypercoagulable TEG results offered predictive protection against bleeding. Current literature shows that normal TEG curve does not exclude defects in the haemostatic process, but also in cases of patients with more complex disturbances of haemostasis, TEG is likely to disclose hypercoagulability.

ITEG failed to predict the hemorrhagic tendency of patients who underwent major cardiac surgery.

The main limitation of this study is the limited number of patients included. However the failure of TEG to predict major bleeding complications was quite obvious, although further research is needed.

\begin{tabular}{|l|l|l|}
\hline Hemorrhagic Status & Pre-anesthesia TEG & Pre-ICU TEG \\
\hline Normal & $60(12)$ & $25(5)$ \\
\hline Hypercoagulable & $40(8)$ & $65(13)$ \\
\hline
\end{tabular}

Results are presented as \% percentages

( $n=$ number of patients)

\section{Conclusion}

Perioperative regular TEG is not helpful in the assessment of bleeding risk. TEG could be used as a tool in complex coagulopathy, complicated cardiac surgical procedures, ongoing hemorrhage, which require extensive blood products utilization. However, the presence of an experienced anaesthesiologist with knowledge of hemostasis, anticoagulation and transfusion medicine leads an important role in the perioperative management of the bleeding patients especially in cardiac surgery.

\section{References:}

1)Faraoni $D$, Willems $A$, Melot $C$, De Hert $S$, Van der Linden $P$. Efficacy of tranexamic acid in paediatric cardiac surgery: a systematic review and meta-analysis. Eur $J$ Cardiothorac Surg 2012; 42: 781-6.

2) Sulman Rafiq, Pär Ingemar Johansson, Sisse Rye Ostrowski, Trine Stissing, Daniel Andreas Steinbrüchel, Hypercoagulability in patients undergoing coronary artery bypass grafting: prevalence, patient characteristics and postoperative outcome European Journal of Cardio-Thoracic Surgery 41 (2012) 550-555.

3) Håkon Reikvam , Egil Steien, Bjørn Hauge , Knut Liseth , Kristin Gjerde Hagen, Rolf Størkson, Tor Hervig, Thromboelastography Transfusion and Apheresis Science 40 (2009) 119-123. 